\title{
Malaria infection diagnosis: comparison between traditional methods and a new freeze-dried PCR multiplex test
}

\author{
Alessia Moiana', Maurizio Gramegna', Fiorella Andrea Solari², Simonetta Gatti ${ }^{2}$ \\ I Sentinel CH SpA, Milano \\ 2 Laboratorio di Parassitologia, Medicina di Laboratorio - Virologia e Microbiologia, Fondazione IRCCS Policlinico San \\ Matteo, Pavia
}

Keywords: Malaria, Plasmodium, early diagnosis, PCR, Freeze drying

Diagnosi dell'infezione malarica: confronto tra metodi tradizionali e un nuovo test in PCR multiplex in formato liofilo

\section{SUMMARY}

Malaria is the most diffused parasitic disease that affects humans. A rapid and accurate diagnosis is a prerequisite for an effective treatment. The development of a new ready-to-use test for the screening and the typing of malaria solve the temperature storage requests of common molecular tests and PCR reagents.

The performances of STAT-NAT Malaria Screening and STAT-NAT Malaria Typing kits were tested on samples obtained from patients suspected to be infected by malaria's parasites, Immigrants (I) and International Travellers (IT), and were compared with the gold standard method (microscopy). Samples of peripheral blood of 35 patients (25 IT and IO I) obtained from San Matteo Hospital in Pavia were analysed. The microscopy identified I8 of them as positive samples: I I samples were positive for Plasmodium falciparum (5 IT, 6 I), 3 for Plasmodium vivax (3 IT), 2 for Plasmodium ovale (II, I IT) and 2 for Plasmodium malariae (2 I). No co-infections were found. STAT-NAT Malaria kits confirmed the results of 18 positive samples as well as of 17 negative samples (100\% sensitivity). In a case STAT-NAT Malaria Typing kit identified the presence of Plasmodium ovale in a sample instead of Plasmodium vivax, as previously diagnosed with the microscopic analysis. The epidemiological analysis and a new microscopic diagnosis confirmed the infection as caused by Plasmodium ovale species ( $100 \%$ specificity).

We conclude that the association of the freeze-dried STAT-NAT Malaria tests could be determinant for the correct detection and typing of malaria, mainly where the temperature controlled storage could be problematic.

\section{INTRODUZIONE}

La malaria è una malattia causata dai parassiti del genere Plasmodium, che sono trasmessi da uomo a uomo attraverso il morso delle zanzare infette. Se non trattata prontamente con farmaci efficaci, la malaria è spesso fatale (1).

Circa 3.3 miliardi di persone - metà della popolazione mondiale - sono a rischio di contrarre la malaria. Nel 2010 si sono registrati circa 216 milioni di casi di malaria e quasi 655000 morti (7). Le persone che vivono nelle aree più povere sono le più vulnerabili.

Uno su cinque $(20 \%)$ di tutti i decessi infantili in Africa è dovuto alla malaria. Si stima che un bambino africano abbia in media da 1.6 a 5.4 episodi di febbre malarica ogni anno. Circa ogni 30 secondi in Africa un bambino muore a causa della malaria (7).

Una diagnosi precoce e un trattamento tempestivo sono due elementi fondamentali nel controllo della malaria. Il trattamento precoce ed efficace della malaria può ridurre la durata dell'infezione e prevenire ulteriori complicazioni tra cui la grande maggioranza dei decessi.

L'accesso al trattamento della malattia deve essere vista non solo come componente di controllo della malaria, ma un diritto fondamentale di tutte le popolazioni a rischio.

Le donne in gravidanza sono ad alto rischio non solo di morte per le complicazioni della malaria grave, ma anche di aborto spontaneo, parto prematuro o nascita del figlio morto.

La malaria è anche una causa di grave anemia materna ed è responsabile di circa un terzo dei bambini con basso peso alla nascita prevenibili. La malaria contribuisce alla morte di circa 10000 donne in gravidanza e fino a 200000 bambini ogni anno nella sola Africa.

La malaria causa una perdita media dell' $1.3 \%$ annuo di crescita economica nei paesi con trasmissione intensa.

Intrappola le famiglie e le comunità in una spirale

\section{Corresponding author: Alessia Moiana}

Sentinel CH. SpA, Via Robert Koch, 2 - 20152 Milano (MI)

Telefono: +39023455 I494 - Fax: +39023455I464

E-mail: alessiamoiana@sentinel.it 
di povertà, colpisce soprattutto gli emarginati e i poveri che non possono permettersi un trattamento o che hanno accesso limitato alle cure sanitarie. La malaria determina 1'aumento della povertà $\mathrm{e}$ difficoltà di apprendimento, riduce infatti la frequentazione delle scuole e dei luoghi di lavoro. Tuttavia, è prevenibile e curabile.

Il metodo gold standard per la diagnosi della malaria è l'esame microscopico di uno striscio di sangue; tuttavia questa tecnica può risultare di bassa sensibilità nei pazienti che presentano basse parassitemie (7).

Inoltre, l'analisi dello striscio di sangue può indurre l'operatore ad una diagnosi errata di co-infezione quando una delle specie, di solito Plasmodium falciparum, è presente in grande quantità e copre così la seconda infezione.

Sebbene la microscopia sia adeguata ai fini della maggior parte delle indagini epidemiologiche, il numero di campioni coinvolti, così come il desiderio di avere un'alta sensibilità di rilevazione e un'identificazione accurata, hanno spinto i ricercatori a sperimentare metodi alternativi $(2,3,5,6)$.

Lo sfruttamento di tecniche di biologia molecolare è culminato nell'uso della reazione a catena della polimerasi (PCR) (4), che rappresenta potenzialmente il metodo più sensibile per la rilevazione specifica di una particolare sequenza di DNA in un dato campione, dato che teoricamente una sola copia della sequenza bersaglio deve essere presente nel campione per un'amplificazione di successo.

La limitazione è, fino ad ora, la richiesta di tecnici qualificati e le esigenze di conservazione a temperatura controllata per questi sistemi.

La disponibilità di test pronti all'uso e conservabili a temperatura ambiente può essere molto importante in alcune zone, per risolvere il problema critico di conservazione in ambiente refrigerato dei kit.

L'introduzione dei test STAT-NAT (Sentinel CH $\mathrm{SpA}$ ) è in grado di aprire una nuova opportunità per lo screening e la tipizzazione della malaria nei paesi in via di sviluppo.

Lo scopo del presente studio è quello di introdurre un nuovo saggio basato sulla PCR multiplex per lo screening e la tipizzazione dell'infezione malarica. Il sistema deve essere in grado di rilevare la presenza di una delle quattro specie del genere Plasmodium che possono colpire l'uomo.

Deve essere inoltre in grado di discriminare tra le quattro specie con la stessa sensibilità e di rilevare tutte le specie presenti in un campione anche in presenza di co-infezioni.

Il sistema contiene un controllo interno per accertare la funzionalità del test, e per escludere l'inibizione della reazione di PCR.

\section{MATERIALI E METODI}

Il saggio è costituito da due test pronti all'uso che contengono tutti gli elementi necessari per l'amplificazione degli acidi nucleici target.

L'utilizzo dei kit STAT-NAT Malaria Screening e Typing è previsto per campioni di sangue intero. Dai campioni di sangue intero è stato estratto il DNA con un protocollo in grado di dare DNA di buona qualità. Per questo studio abbiamo utilizzato un sistema a base di silice sviluppato internamente. Il protocollo di estrazione inizia con una fase di lavaggio per lisare i globuli rossi ed eliminare la presenza di emoglobina. Poi una soluzione contenente un agente caotropico e una sospensione di silice per il legame del DNA sono aggiunte al campione.

La sospensione è lasciata 3-5 minuti a temperatura ambiente. Un ulteriore lavaggio con etanolo completa la procedura. Il pellet è lasciato asciugare per cinque minuti a temperatura ambiente e poi disciolto in 100-200 $\mu 1$ di acqua sterile.

Il DNA viene immediatamente utilizzato per il protocollo di PCR e poi conservato a $-20^{\circ} \mathrm{C}$.

I due test pronti all'uso includono tutti gli elementi necessari per l'amplificazione degli acidi nucleici target. In ciascuno dei due test, uno per lo screening e il rilevamento della presenza del Plasmodium falciparum (Figura I), l'altro per la tipizzazione delle altre tre specie che infettano l'uomo (Plasmodium vivax, Plasmodium ovale e Plasmodium malariae; Figura II), è stato incluso un controllo interno. I cinque set di primer disegnati nella regione del gene 18S RNA ribosomale (18S rRNA) (uno per lo screening e quattro per ciascuna delle quattro specie), sono stati testati su ceppi di riferimento ottenuti dal Policlinico San Matteo. Questi primers danno origine a frammenti di 169 bp (Plasmodium falciparum), 118 bp (Plasmodium ovale), 95 bp (Plasmodium vivax), e 106 bp (Plasmodium malariae).

Il set di primer per lo screening si basa su sequenze degenerate ed è in grado di rilevare tutte e quattro le specie di Plasmodium. La dimensione del frammento amplificato dipende dalla specie e varia tra 220 e $240 \mathrm{bp}$.

In ogni provetta è stato inoltre incluso un controllo interno, sia come controllo del sistema di estrazione che come controllo dell'efficienza di amplificazione. I primers per il controllo interno sono stati disegnati all'interno del gene della beta globina umana, e danno un amplificato di $268 \mathrm{bp}$ (Figura I; Figura II).

Tutti i campioni sono stati precedentemente diagnosticati con i metodi gold standard utilizzati negli ospedali (microscopia su striscio di sangue e saggi immunocromatografici), e poi testati in cieco in PCR. 
In Tabella 1 e Tabella 2 sono riassunte rispettivamente la distribuzione dei campioni e la distribuzione dei campioni positivi; in Tabella 3 è mostrato il protocollo di amplificazione ed il profilo termico della PCR.

\section{RISULTATI}

Il pannello dei campioni considerati nel presente studio comprende forti positivi, così come deboli positivi e negativi. Tutti i campioni sono stati testati ripetutamente per la valutazione delle prestazioni intra ed inter-saggio.

I risultati qualitativi ottenuti utilizzando i kit STAT-NAT Malaria screening e STAT-NAT Malaria Typing sono stati confrontati con i risultati attesi per il pannello analizzato. I risultati hanno mostrato un perfetto accordo in tutti i campioni del pannello.

Anche in presenza di livelli molto bassi di infezioni parassitarie (5 parassiti $/ \mathrm{ml}$ in alcuni campioni), il test è stato in grado di rilevare la presenza di plasmodi nel campione. La Figura III mostra il profilo elettroforetico di una corsa post-PCR ottenuta con il kit STAT-NAT Malaria Screening.

La Figura IV mostra il profilo elettroforetico di una corsa post-PCR ottenuta con il kit STAT-NAT Malaria Typing. Tutte le bande corrispondenti al controllo interno (beta globina) sono chiaramente amplificate. In un caso il kit STAT-NAT Malaria Typing ha identificato un campione come positivo per Plasmodium ovale mentre dalla microscopia risultava positivo per Plasmodium vivax.

L'analisi retrospettiva del campione ha confermato i risultati della PCR. In un secondo caso siamo stati in grado di seguire l'infezione durante il trattamento farmacologico del paziente. Campioni di sangue intero sono stati prelevati dal paziente al tempo zero, dopo 72 ore, dopo 10 e 20 giorni.

$\mathrm{Se}$ al microscopio la diagnosi è risultata negativa dopo 72 ore, la PCR ha evidenziato una positività fino a 20 giorni. La Figura $V$ mostra il profilo di amplificazione durante il follow-up del trattamento del paziente. Al fine di valutare la specificità del test abbiamo utilizzato anche campioni positivi per le altre infezioni parassitarie.

Non sono stati osservati falsi positivi in tutti $i$ campioni controllati (dati non riportati).

La specificità del test è risultata pari al 100\%.

La sensibilità del test basata sul gold standard è risultata pari al $94 \%$, ma arriva al $100 \%$ dopo la diagnosi retrospettiva. Il formato pronto all'uso del test evita problemi legati alla cross-contaminazione, dovuta alla comune preparazione della miscela di reazione di PCR che prevede molteplici step.

\section{DISCUSSIONE}

Il saggio liofilo e pronto all'uso qui descritto è in grado di rilevare tutte le infezioni malariche di un pannello di riferimento e di identificare tutti i campioni negativi senza cross-contaminazioni o falsi positivi.

Il formato liofilo permette di aumentare la quantità di DNA estratto da aggiungere alla miscela di reazione di PCR fino a $25 \mu \mathrm{L}$, accrescendo la sensibilità di diverse volte.

Il saggio, come utilizzato in questo studio, mostra una sensibilità e una specificità del $100 \%$ per l'identificazione dell'infezione da Plasmodium.

La conservazione a temperatura ambiente fino ad un anno del sistema è una caratteristica molto importante da considerare quando uno screening per l'infezione malarica deve essere attuato nei Paesi dove tale infezione è endemica, in genere Paesi in via di sviluppo delle regioni tropicali e sub-tropicali.

Questo saggio può essere considerato uno strumento importante per la diagnosi di soggetti sospettati di essere infetti, e per la prevenzione della popolazione a rischio.

Infine, il formato del saggio ne permette una potenziale applicazione in test in PCR in completa automazione.

Tabella I. Distribuzione dei campioni.

\begin{tabular}{lccc}
\hline & $\begin{array}{c}\text { Viaggiatori } \\
\text { internazionali }\end{array}$ & Immigrati & Totale \\
\hline Positivo & 9 & 9 & 18 \\
\hline Negativo & 16 & $\mathrm{I}$ & 17 \\
\hline Totale & 25 & 10 & 35 \\
\hline
\end{tabular}

Tabella 2. Distribuzione dei campioni positivi.

\begin{tabular}{llcc}
\hline \multicolumn{1}{c}{$\begin{array}{c}\text { Viaggiatori } \\
\text { internazionali }\end{array}$} & Immigrati & Totale \\
$\begin{array}{l}\text { Plasmodium. } \\
\text { falciparum }\end{array}$ & 5 & 6 & $\mathrm{II}$ \\
\hline $\begin{array}{l}\text { Plasmodium vivax } \\
\text { Plasmodium ovale }\end{array}$ & 3 & - & 3 \\
\hline $\begin{array}{l}\text { Plasmodium } \\
\text { malariae }\end{array}$ & 2 & $\mathrm{I}$ & 2 \\
\hline Totale & $\mathrm{II}$ & - & 2 \\
\hline
\end{tabular}

Tabella 3. Protocollo di PCR. Sono descritti il profilo termico e le tempistiche del protocollo di amplificazione.

\begin{tabular}{cccc}
\hline N. di cicli & denaturazione & annealing & estensione \\
\hline 40 & $\mathrm{I}$ & $94^{\circ} \mathrm{C}-2 \mathrm{~min}$ & \\
\hline $\mathrm{I}$ & $94^{\circ} \mathrm{C}-30 \mathrm{sec}$ & $55^{\circ} \mathrm{C}-30 \mathrm{sec}$ & $72^{\circ} \mathrm{C}-45 \mathrm{sec}$ \\
\hline & & & $72^{\circ} \mathrm{C}-5 \mathrm{~min}$ \\
\hline
\end{tabular}




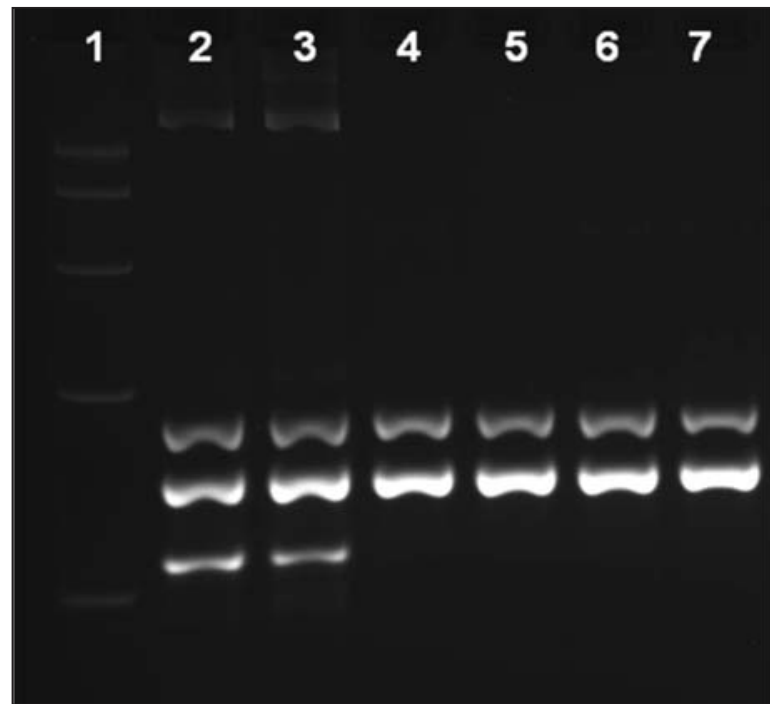

Figura I. STAT-NAT Malaria Screening. Corsia I: BenchTop PCR Markers (Promega), 2-3: campioni positivi per Plasmodium falciparum, 4: campione positivo per Plasmodium malariae, 5: campione positivo per Plasmodium vivax, 6-7: campioni positivi per Plasmodium ovale. La banda superiore corrisponde al controllo interno.

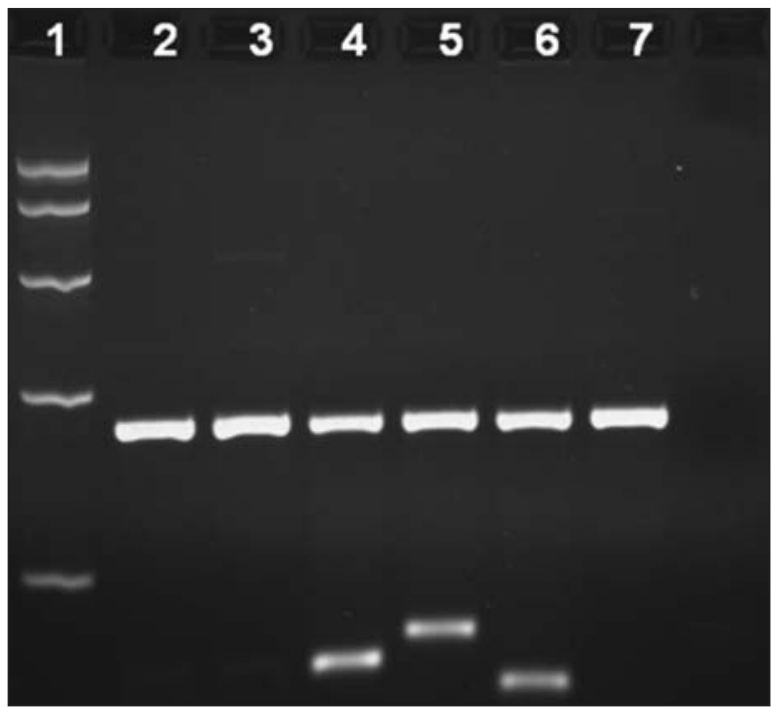

Figura II. STAT-NAT Malaria Typing. Corsia I: BenchTop PCR Markers (Promega), 2-3 and 7: campioni negativi, 4: campione positivo per Plasmodium malariae, 5: campione positivo per Plasmodium ovale, 6: campione positivo per Plasmodium vivax. La banda superiore corrisponde al controllo interno.

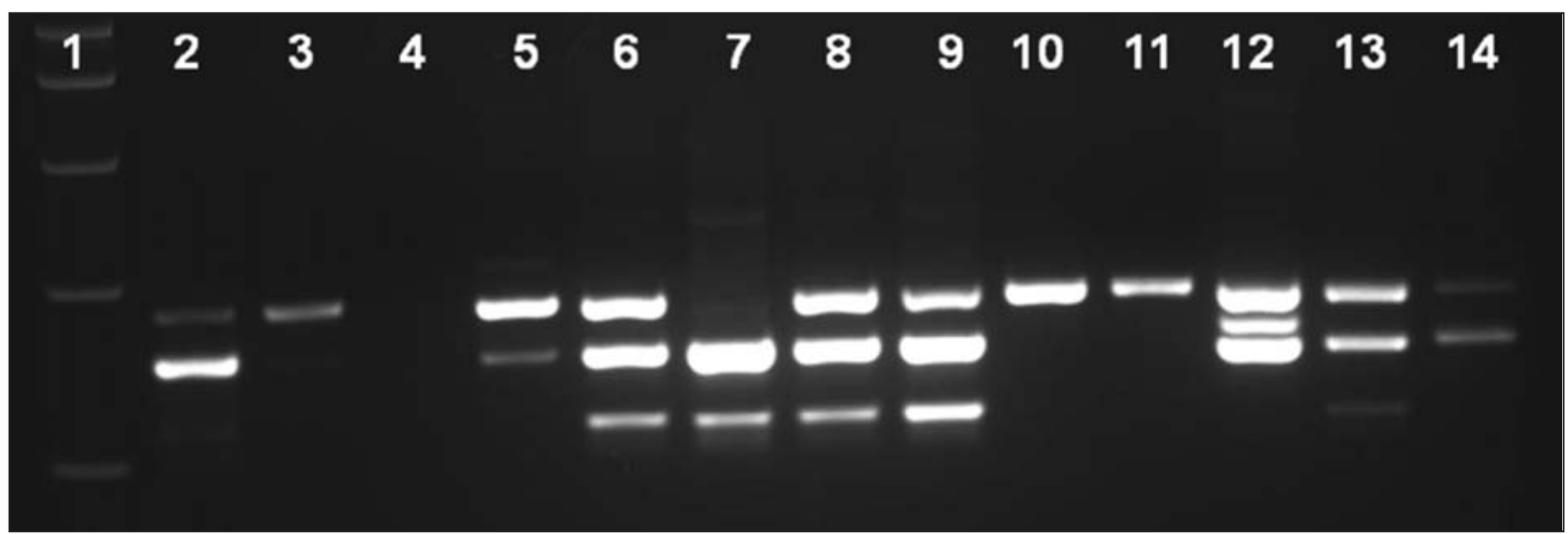

Figura III. Utilizzo di STAT-NAT Malaria Screening con campioni biologici ignoti. Corsia I BenchTop PCR Markers (Promega), corsie 2, 3, 5- I4: campioni ignoti analizzati con il kit STAT-NAT Malaria Screening, corsia 4: NTC, no template control.

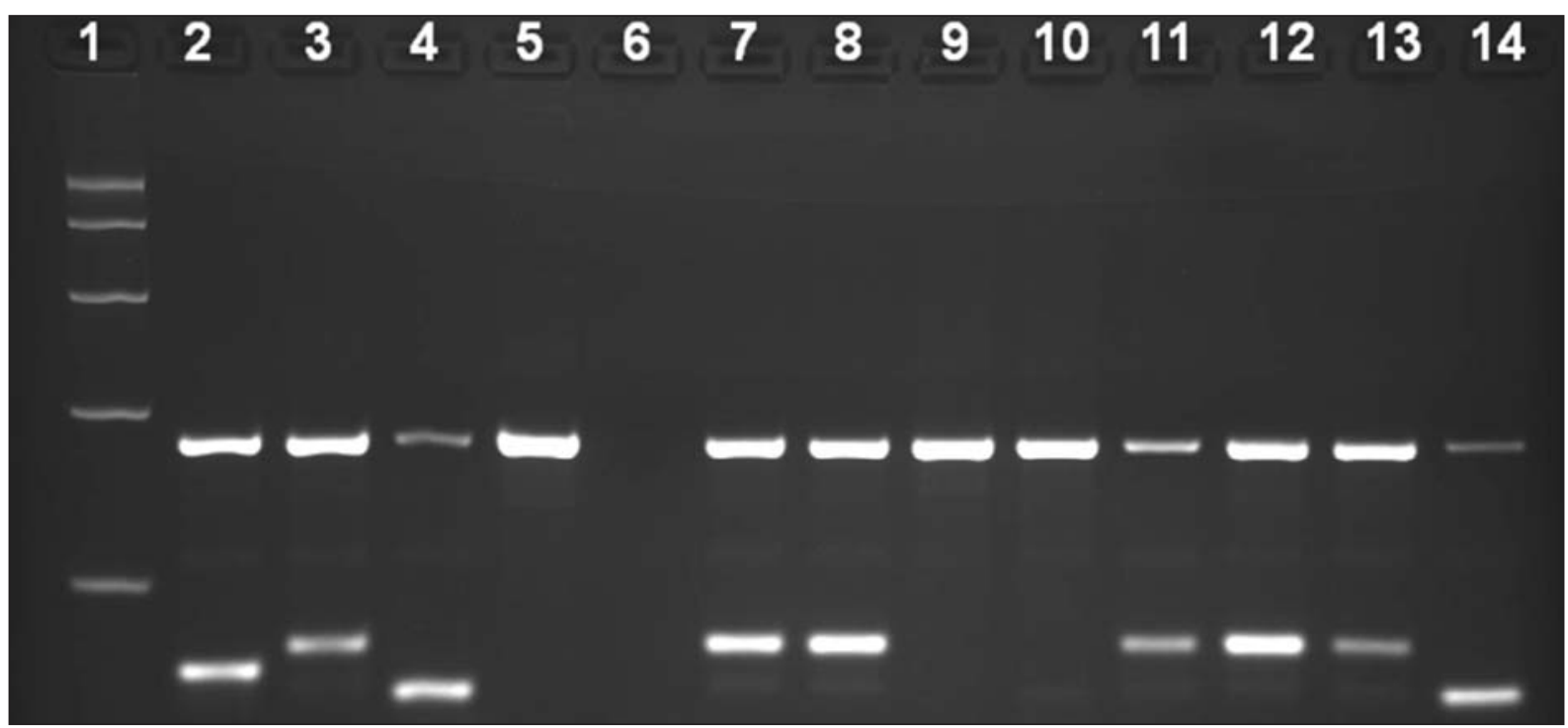

Figura IV. Utilizzo di STAT-NAT Malaria Typing con campioni biologici ignoti. Corsia I: BenchTop PCR Markers (Promega), corsie 2-5, 7-I4: campioni ignoti analizzati con il kit STAT-NAT Malaria Typing, corsia 6: NTC, no template control. 


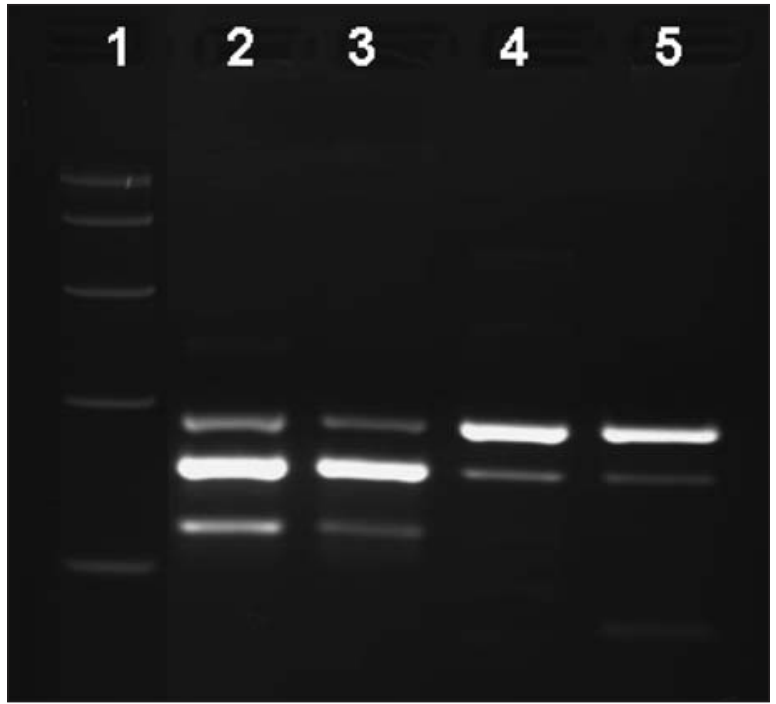

Figura V. Utilizzo di STAT-NAT Malaria Screening durante il follow-up di un paziente. Corsia I: BenchTop PCR Markers (Promega), corsia 2: tempo zero, corsia 3: 72 ore dal primo prelievo, corsia 4: 10 giorni dal primo prelievo, corsia 5: 20 giorni dal primo prelievo.

\section{BIBLIOGRAFIA}

1. Bisoffi Z, Marocco S, Monteiro G. Malaria. In: Parassiti e parassitosi umane: dalla clinica al laboratorio (a cura di Scaglia M, Gatti S, Rondanelli EG). Selecta Medica Publ, Pavia, 2006; 3: 23-58.

2. Gatti S, Gramegna M, Bisoffi Z, et al. The Gispi Study Group. A comparison of three diagnostic techniques for malaria: a rapid diagnostic test (NOW Malaria), PCR and microscopy. Ann Trop Med Parasitol, 2007; 101(3): 195-204.

3. Mixson-Hayden T, Lucchi NW, Udhayakumar V. Evaluation of three PCR-based diagnostic assays for detecting mixed Plasmodium infections. BMC Research Notes, 2010; 3: 88-94.

4. Mullis KB, Faloona FA. Specific synthesis of DNA in vitro via a polymerase-catalyzed chain reaction. Methods Enzymol, 1987; 155: 335-50.

5. Myjak P, Nahorski W, Pieniazek NJ, Pietkiewicz H. Usefulness of PCR for diagnosis of imported malaria in Poland. Eur J Clin Microbiol Infect Dis, 2002; 21: 215-8.

6. Ochola LB, Vounatsou P, Smith T, Mabaso ML, Newton CR. The reliability of diagnostic techniques in the diagnosis and management of malaria in the absence of a gold standard. Lancet Infect Dis, 2006; 6(9): 582-8.

7. WHO, World Malaria Report, 2011 Geneva, Switzerland. 